\title{
Significados personales del concepto de juego equitativo en niños y niñas costarricenses
}

\section{Personal meanings of the concept of fair play in Costa Rican boys and girls Significados pessoais do conceito de jogo equitativo em crianças costarriquenhas}

\author{
Luis Armando Hernández-Solís \\ Universidad Estatal a Distancia \\ San José, Costa Rica \\ Ihernandez@uned.ac.cr \\ (D) https://orcid.org/0000-0003-2956-8102 \\ Carmen Batanero \\ Universidad de Granada \\ Granada, España \\ batanero@ugr.es \\ (iD https://orcid.org/0000-0002-4189-7139 \\ Rocío Álvarez-Arroyo \\ Universidad de Granada \\ Granada, España \\ rocioaarroyo@ugr.es \\ (D) https://orcid.org/0000-0002-3201-8542 \\ María M. Gea \\ Universidad de Granada \\ Granada, España \\ mmgea@ugr.es \\ (D) https://orcid.org/0000-0002-5229-0121
}

Recibido - Received: 22 / 02 / 2020 Corregido - Revised: 20 / 04 / 2020 Aceptado - Accepted: 30/ 04 / 2020

DOl: https://doi.org/10.22458/ie.v23i34.3429

URL: https://revistas.uned.ac.cr/index.php/innovaciones/article/view/3429

\begin{abstract}
Resumen: El objetivo de este trabajo es describir los significados personales que asigna al juego equitativo el estudiantado costarricense de sexto curso de educación primaria. A partir de un análisis cuantitativo y cualitativo de las respuestas a dos problemas asociados a la noción de juego equitativo, se describen las estrategias empleadas y conflictos semióticos detectados; también, se comparan los resultados obtenidos con los que se establecieron en la investigación previa. En general, se muestra una adecuada comprensión de la noción de juego equitativo; sin embargo, presentan grandes dificultades al justificar la ganancia según la esperanza de ganar de cada jugador y con frecuencia justifican sus respuestas con estrategias aditivas, las cuales generan respuestas incorrectas. Además, se obtuvo un rendimiento inferior respecto a los resultados consignados en estudios previos con sujetos de igual edad que no recibieron enseñanza en el tema.
\end{abstract}

Palabras Clave: Comprensión, Intuición, Probabilidad, Juego, Evaluación, Matemática, Educación 
Summary: This paper aims to describe the personal meanings assigned to the term fair play by Costa Rican sixth-grade students. Deriving out of a quantitative and qualitative analysis of the answers to the problems associated with the notion of fair play, we describe the strategies used and the semiotic conflicts detected. Also, the obtained outcomes are compared with data gathered in previous research. In general, this paper shows an adequate understanding of the notion of fair play. However, a significant difficulty to justify winning according to each player's hope of winning was found; they often justified their answers with additive strategies, which generated incorrect answers. Furthermore, a lower performance was obtained concerning the results reported in previous studies with subjects of the same age who did not receive education in the subject.

Keywords: comprehension, probability, games, evaluation, Mathematics, Education

Resumo: $O$ objetivo deste trabalho é descrever os significados pessoais atribuídos aos alunos costarriquenhos do sexto ano do ensino fundamental. A partir de uma análise quantitativa e qualitativa das respostas a dois problemas associados à noção de jogo equitativo "fair play", são descritas as estratégias empregadas e os conflitos semióticos detectados; também, os resultados obtidos são comparados com aqueles estabelecidos em pesquisas anteriores. Em geral, é demonstrado um entendimento adequado da noção de fair play; no entanto, eles apresentam grandes dificuldades ao justificar a vitória de acordo com a esperança de vitória de cada jogador e frequentemente justificam suas respostas com estratégias aditivas, que geram respostas incorretas. Além disso, foi obtido um desempenho inferior em relação aos resultados relatados em estudos anteriores com sujeitos da mesma idade que não receberam instrução no assunto.

Palavras chave: compreensão, intuição, probabilidade, jogo, avaliação; matemática, educação

\section{INTRODUCCIÓN}

Como consecuencia del desarrollo de la inferencia estadística y el incremento de experimentos aleatorizados en muchas investigaciones, la probabilidad ha adquirido relevancia en distintas áreas profesionales y científicas. Además, la probabilidad es una herramienta necesaria para la toma de decisiones en condiciones aleatorias (Batanero, 2005). Pino y Estrella (2012) señalan que su estudio tiene un alto valor educativo; pues muestra las limitaciones de argumentos puramente deductivos. Debido a estas razones, se indica que los conocimientos probabilísticos deben formar parte de los objetivos de alfabetización matemática para los ciudadanos (Jones, Langrall y Mooney, 2007; Nilsson y Li, 2015).

Esta importancia, señalada en el párrafo anterior, ha repercutido en los currículos de matemáticas de varios países, en los cuales se introducen contenidos de probabilidad desde edades tempranas (por ejemplo, Australian Curriculum, Assessment and Reporting Authority, 2013; Ministerio de Educación, Cultura y Deporte, 2014; National Council of Teachers of Mathematics, 2000). Costa Rica no ha sido la excepción y en el 2012 se aprobaron nuevos Programas de Estudio de Matemática para la educación primaria y secundaria (MEP, 2012), se le dio gran relevancia al área de Estadística y Probabilidad, cuyos conocimientos y expectativas de aprendizaje se organizan de manera integrada, desde el primer año de educación primaria hasta el último de educación secundaria.

Este trabajo se centra en los juegos de azar, práctica común en todas las civilizaciones y desde su origen estuvieron asociados a ideas intuitivas sobre la esperanza matemática y el juego equitativo. De hecho, el cálculo de probabilidades se origina por la necesidad del jugador de valorar de antemano sus posibles pérdidas o ganancias (Batanero, Henry y Parzysz, 2005). Este comienzo histórico de la teoría en probabilidad tiene un gran impacto en su didáctica; pues los juegos de azar son también uno de los principales contextos en donde los niños y las niñas empiezan a tomar conciencia de lo impredecible de sus resultados y surge su interés por realizar estimaciones probabilísticas, incluso antes de la instrucción (Batanero, Ortiz, Gómez y Gea, 2019; Peard, 1990).

Además, el interés de los juegos de azar como recurso de aprendizaje se basa en que permiten reforzar parte de las ideas fundamentales que Gal (2005) incluye en su concepto de alfabetización probabilística, en concreto las de aleatoriedad, variabilidad, incertidumbre e independencia. Requiere, asimismo, estimar o calcular probabilidades y hacer uso del lenguaje de probabilidad. 
En relación con los antecedentes, a pesar de su importancia, son todavía escasas las investigaciones sobre las intuiciones que las personas tienen sobre la idea de juego equitativo, comparado con estudios de otras nociones probabilísticas básicas. Algunas de ellas estudian sus estrategias para decidir si un juego es o no equitativo (Watson y Collis, 1994), intuiciones sobre la esperanza matemática (Schlottmann y Anderson, 1994) y la influencia de las experiencias extraescolares en el desarrollo de la idea de equitatividad y su relación con la de probabilidad (Lidster, Watson, Collis y Pereira-Mendoza, 1996).

Más asociado al estudio se encuentra la investigación realizada por Cañizares, Batanero, Serrano y Ortiz (1999), quienes analizaron la comprensión de la idea de juego equitativo de los niños y las niñas entre 10 y 14 años, relacionándola con sus creencias en el terreno de la probabilidad y su influencia en la asignación de probabilidades. Los autores indican que algunas personas muestran ideas de este tipo como: a) introducir factores externos, tales como "hacer trampas" en sus argumentos; b) igualar las ganancias en juegos donde los participantes no tienen las mismas probabilidades; c) exigir que haya un alto número de partidas para que el juego sea equitativo, ignorando así la independencia de los ensayos; y d) considerar que todos los juegos aleatorios, si no se hace trampa, son equitativos.

Por otro lado, algunas investigaciones referentes al conocimiento profesional del profesor sobre las nociones de probabilidad (Azcárate, 1995; Mohamed y Ortiz, 2012; Ortiz, Batanero y Contreras, 2012) han encontrado, en docentes y profesores en formación, dificultades para identificar los juegos equitativos; pues los participantes basan sus argumentos en la equiprobabilidad de los resultados, reglas aritméticas o argumentación combinatoria incorrecta.

También se tienen en cuenta algunos estudios como los de Fischbein y Gazit (1984), quienes exploraron factores que podían afectar los juicios probabilísticos en niños(as) y adolescentes, por ejemplo, la influencia de supersticiones como "empezar con el pie derecho" y otras creencias populares. Asimismo, se considera el estudio de Green (1982), el cual replica en versión papel y lápiz algunos experimentos de Piaget y trató de determinar el nivel de razonamiento probabilístico de los sujetos de su muestra, con ello obtuvo un paralelismo con las etapas de desarrollo de este razonamiento descritas por Piaget e Inhelder (1951).

Otras personas podrían suponer que todos los sucesos aleatorios son equiprobables por naturaleza; es decir, generalizan indebidamente la regla de Laplace ("sesgo de equiprobabilidad", Lecoutre, 1992). Cañizares (1997) encontró en los argumentos de los participantes creencias subjetivas, como no reconocer la independencia en contextos de loterías y la búsqueda de explicaciones causales que afecten la probabilidad de los sucesos (por ejemplo, el color favorito o la edad de un niño); igualmente identificó argumentos asociados a consideraciones físicas como por ejemplo la disposición de las bolas en la imagen.

Por su lado, en cuanto a los referentes teóricos del trabajo, el estudio se basa en el Enfoque Ontosemiótico (EOS) del conocimiento y la instrucción matemática (Godino y Batanero, 1994; Godino, Batanero y Font, 2007; 2019). El EOS asume una formulación ontológica de los objetos matemáticos, cuya noción primitiva es la de situación-problema, a partir de la cual se definen los conceptos teóricos de práctica, objeto (personal e institucional) y significado (Godino, Batanero y Font, 2007; 2019). Una práctica es "toda actuación o manifestación (lingüística o no) realizada por alguien para resolver problemas matemáticos, comunicar a otros la solución, validar la solución y generalizarla a otros contextos y problemas" (Godino y Batanero, 1994, p. 334). En este marco teórico se distingue entre las prácticas realizadas por cada persona (prácticas personales) y las normadas por los sujetos de una institución (prácticas institucionales); por ejemplo: la institución escolar. Es decir, dentro de la institución se realizan prácticas sociales y se toman en cuenta instrumentos, reglas y modos de funcionamiento (Godino y Batanero, 1994).

En este estudio se pretende analizar las prácticas matemáticas realizadas por la muestra de estudiantes al resolver dos problemas asociados a la noción de juego equitativo y se compara con las prácticas regladas por la institución escolar. 
En el EOS (Godino y Batanero, 1994; Godino, Batanero y Font, 2007; 2019) se considera un objeto matemático como emergente del sistema de prácticas asociadas a un campo de problemas y dicho sistema de prácticas confiere el significado al objeto. Según si se considera el sistema de prácticas realizado dentro de una institución o específico de una persona, se hablará de significado institucional o personal del objeto. La intersección entre estos dos sistemas de prácticas sería lo que la persona "conoce" o "comprende" del objeto, desde el punto de vista de la institución. Por otro lado, las prácticas que no corresponden con las regladas por la institución se denotan como conflictos semióticos, los cuales se producen cuando la persona asigna al objeto un significado que no concuerda con los considerados como correctos, desde el punto de vista institucional. Además, cabe señalar que la comprensión alcanzada por un sujeto en un momento dado no puede considerarse como una variable dicotómica (todo o nada); sino que podría haber alcances conceptuales parciales de la comprensión de un objeto matemático.

Esta dualidad en el significado de un objeto matemático (institucional y personal) permite abordar la evaluación de los conocimientos (inobservables) de las personas por medio de sus prácticas al resolver problemas (observables), las cuales se pueden considerar indicadores empíricos de dichos conocimientos o sus conflictos semióticos. En esta premisa se fundamenta el estudio, de modo que, para analizar el significado personal sobre el juego equitativo de cada sujeto de la muestra, se interpretará el conjunto de prácticas observables mostradas durante la resolución de los problemas propuestos.

Al realizar o evaluar una práctica matemática asociada a una situación-problema, resulta necesario interpretar los lenguajes (verbales, tabulares, gráficos y simbólicos) que representan a otros objetos matemáticos (conceptos, procedimientos, proposiciones y argumentos) que intervienen en su resolución. Estos objetos se denominan primarios (Godino, Batanero y Font, 2007) y se relacionan entre sí formando configuraciones, las cuales se comprenden como redes de objetos que intervienen o emergen de los sistemas de prácticas. De este modo, las configuraciones pueden ser epistémicas (referidas a redes de objetos institucionales) o cognitivas (referidas a redes de objetos personales).

Otro referente teórico es la idea de intuición, de acuerdo con Fischbein (1987), quien la considera parte de la conducta inteligente e interviene en las acciones y el razonamiento de un individuo. Define el conocimiento intuitivo como "una clase de conocimiento que no está basado en la evidencia empírica o en argumentos lógicos rigurosos y, a pesar de ello, se tiende a aceptarlo como cierto y evidente" (Fischbein, 1987, p. 26). Lo considera un tipo de cognición caracterizada por la auto-evidencia; pues las afirmaciones del individuo poseen certeza intrínseca, incluso de carácter implícito, que perseveran en sus decisiones y se imponen de modo coercitivo como si se tratara de una teoría capaz de extrapolarse, con carácter global; por lo tanto, se diferencia de la mera percepción, ya que abarca más allá de los hechos. Sus características se describen en la Figura 1.

No deben confundirse las intuiciones con las creencias, pues no comparten las características de globalidad, carácter implícito, categoría teórica o extrapolatoria. En este trabajo se comprenden como creencias las ideas firmemente sostenidas y con frecuencia erróneas del estudiantado.

Fischbein (1987) diferencia entre intuiciones primarias, las cuales se adquieren en forma directa con la experiencia y sin necesidad de instrucción sistemática, e intuiciones secundarias, que se forman como consecuencia de la educación. Fischbein (1975) señala además que una intuición secundaria no es una fórmula aprendida de memoria o utilizada automáticamente, sino una convicción; no se forma a partir de la lectura o una explicación teórica, sino de su uso en las acciones y las predicciones a lo largo de la vida. Por ejemplo, una intuición primaria correcta sería la equibrobabilidad entre las dos caras de una moneda (escudo y corona), y una intuición secundaria errónea sería que al lanzar una moneda varias veces y haber obtenido siempre escudo, se piense que en el próximo lanzamiento habría más probabilidad de que saliera corona. 
De acuerdo con Batanero (2005), el significado intuitivo de la probabilidad puede aparecer tanto en niños(as) como en personas sin instrucción en el tema y se puede evidenciar en expresiones coloquiales para cuantificar los sucesos inciertos y así expresar su grado de creencia en ellos. Consecuentemente, aunque no se haya enseñado en forma explícita a los y las estudiantes de la muestra la idea de juego equitativo en las clases de matemática, estos pueden haber desarrollado significados personales, asociados a intuiciones primarias a través de sus juegos.

Figura 1. Características de la intuición probabilística.

\begin{tabular}{|c|c|c|c|c|}
\hline \multirow[b]{2}{*}{$\begin{array}{l}\text { Auto-evidencia } \\
\text { Se realizarán afirmaciones } \\
\text { bajo evidencia; por ejemplo, } \\
\text { si se saca una bola al azar en } \\
\text { una caja con diez bolas } \\
\text { bancas, se indica que el color } \\
\text { será blanco (evento seguro),y } \\
\text { que el evento de sacar bola } \\
\text { negra no se dará (evento } \\
\text { imposible). Se reconocen } \\
\text { estos razonamientos como } \\
\text { verdad sin necesidad de } \\
\text { justificarlos: autoexplicables. }\end{array}$} & \multicolumn{2}{|r|}{ Certeza intrínseca } & Categoria teórica & \\
\hline & \multicolumn{2}{|c|}{$\begin{array}{l}\text { Las intuiciones se aceptan como } \\
\text { ciertas, no solo por ser evidentes, } \\
\text { sino porque se tiene presente } \\
\text { algún ejemplo o argumento } \\
\text { teórico que hace tener confianza. }\end{array}$} & \multirow{2}{*}{$\begin{array}{l}\text { Es una teoría (no percepción o } \\
\text { habilidad) que al aceptarla, se } \\
\text { reconoce la necesidad y } \\
\text { generalidad de ésta. Se capta la } \\
\text { universalidad de un principio o } \\
\text { relacón a través de una realidad } \\
\text { particular modelada en una } \\
\text { representación específica. Por } \\
\text { ejemplo, al afirmar "al lanzar una } \\
\text { moneda al aire cada cara tiene la } \\
\text { misma posibilidad de salir", la } \\
\text { regularidad del objeto hace } \\
\text { pensar en equiprobabilidad, pero } \\
\text { lo que se intuye es la universalidad } \\
\text { de dicha propiedad. }\end{array}$} & \multirow{3}{*}{$\begin{array}{c}\text { Las intuiciones se imponen } \\
\text { subjetivamente sobre el } \\
\text { razonamiento, } \\
\text { considerándose absolutas y } \\
\text { dejando de lado otras } \\
\text { alternativas. Un ejemplo es la } \\
\text { falacia del jugador, donde los } \\
\text { participantes comenzaron a } \\
\text { apostar, por ejemplo, } \\
\text { cantidades sucesivamente } \\
\text { mayores de dinero al rojo si } \\
\text { la bola había caído diez } \\
\text { veces consecutivas en el } \\
\text { negro. Es decir, es difícil } \\
\text { aceptar, intuitivamente, que } \\
\text { despues de una racha larga } \\
\text { de que la bola caíga en } \\
\text { negro, la probabilidad de } \\
\text { que volviera a caer en negro } \\
\text { sea la misma que la de caer } \\
\text { en roja. }\end{array}$} \\
\hline \multirow{2}{*}{\multicolumn{2}{|c|}{$\begin{array}{l}\text { Capacidad extrapolatoria } \\
\text { Excede la información disponible. Un } \\
\text { ejemplo es el salto en el razonamiento } \\
\text { cuando se trata con procesos infinitos o } \\
\text { conjuntos infinitos, por ejemplo, la Ley } \\
\text { de los grandes números. La forma } \\
\text { dinámica del infinito no presenta } \\
\text { dificultad aparente, pareciera que se } \\
\text { está naturalmente capacitado para ello. }\end{array}$}} & conciente de las & & \\
\hline & & $\begin{array}{l}\text { intuiciones; la reacciones } \\
\text { intuitivas son una } \\
\text { estructura superficial } \\
\text { que expresa lo profundo } \\
\text { de los mecanismos y } \\
\text { procesos mentales. }\end{array}$ & $\begin{array}{l}\text { niento } \\
\text { Es una } \\
\text { que ofrece } \\
\text { initaria } \\
\text { t. }\end{array}$ & \\
\hline
\end{tabular}

Fuente: elaboración propia a partir de Fischbein (1987).

En ese sentido, Schlottmann y Anderson (1994) concluyeron que, incluso niños y niñas muy pequeños tienen una intuición correcta sobre la idea de esperanza matemática, cuando tienen en cuenta tanto la probabilidad como el valor del premio para tomar sus decisiones, siendo resultados que se corroboraron en estudios posteriores en juegos de habilidad (p. ej. Bayless y Schlottmann, 2010). En cualquier caso, también se podrían adquirir intuiciones que no coincidan con los significados institucionales asociados a los problemas planteados, y generar errores sistemáticos que evidencian conflictos semióticos.

El currículo costarricense plantea para la educación primaria el análisis de las probabilidades mediante juegos de azar y problemas del contexto estudiantil. Y, una expectativa de aprendizaje es identificar eventos igualmente probables de acuerdo con el número de resultados simples pertenecientes a cada evento (MEP, 2012). Sin embargo, no se hace referencia de manera explícita a situaciones asociadas a la idea de juego equitativo o donde se deba establecer la ganancia si uno de ellos tiene ventaja de acuerdo con la esperanza de ganar de cada jugador. Tampoco se dispone en Costa Rica de investigaciones que evalúen la comprensión del estudiantado de educación primaria sobre este tema, aunque sí se han iniciado estudios de evaluación relacionados con otros contenidos de probabilidad (Hernández-Solís, Batanero, Gea y Álvarez-Arroyo, 2021).

Para contribuir a llenar este vacío, el objetivo de este trabajo es describir los significados personales que el estudiantado costarricense de sexto curso de educación primaria asigna al juego equitativo, e identificar algunos conflictos semióticos, comparando los resultados obtenidos con los establecidos en otras investigaciones previas. 


\section{MATERIALES Y MÉTODO}

El enfoque del trabajo es interpretativo, ya que se centra en comprender un fenómeno educativo (significado personal que asignan los estudiantes al juego equitativo) por medio del análisis de elementos cuantitativos y cualitativos reflejados en las respuestas a un cuestionario (Cerrón, 2019; Gil, León, y Morales, 2017).

Es, además, una investigación aplicada (Bisquerra, 1989); pues busca utilizar los resultados de estudios previos como los de Cañizares (1997), Fischbein y Gazit (1984) y Green (1982), con la finalidad de proporcionar conocimiento que oriente la enseñanza y el aprendizaje de la probabilidad en la educación primaria en el contexto costarricense.

\section{Muestra y problemas propuestos}

La población de interés es el estudiantado de sexto curso de la educación primaria costarricense. La muestra es intencional y estuvo formada por 55 estudiantes (27 niñas y 28 niños) de sexto curso de educación primaria, 40 de once años y 15 de doce años. La educación matemática del estudiantado de la muestra se rige por el programa de estudios de Matemática del Ministerio de Educación Pública de Costa Rica (MEP, 2012).

Mediante entrevistas a las docentes encargadas de los grupos que forman la muestra, se conoce que ambos desarrollaron los contenidos del área de Estadística y Probabilidad del año lectivo anterior a partir de los mismos libros de texto, Santillana (2019a; 2019b), dándole gran relevancia a este recurso y a las actividades de "lápiz y papel". Además, en la revisión de dichos libros no se identificaron problemas o actividades asociadas al juego equitativo o equiparación de ganancias en juegos; en general, había poco desarrollo del tema de probabilidad. Cabe señalar que, al momento de la aplicación del cuestionario, el estudiantado estaba comenzando el curso lectivo (a un mes de haberlo iniciado); por lo tanto, aún no habían estudiado contenidos de probabilidad correspondientes al sexto curso.

A los constituyentes de la muestra se les propusieron dos problemas asociados a la comprensión del juego equitativo, tomados de instrumentos de evaluación utilizados previamente por Green (1982) y Cañizares (1997), quienes analizaron la validez y la fiabilidad de sus instrumentos. En este trabajo no se efectúa un nuevo estudio de fiabilidad y validez al tratarse de problemas validados en forma previa y ser un estudio de carácter exploratorio.

Cada individuo los resolvió por escrito e individualmente, en presencia del profesorado y uno de los autores, los cuales se aseguraron de la correcta comprensión de los enunciados. Los problemas empleados son los siguientes:

Problema 1: Eduardo tiene en su caja 10 bolas blancas y 20 negras. Luis tiene en una caja 30 bolas blancas y 60 negras. Juegan a sacar al mismo tiempo una bola de su caja (con los ojos cerrados) y el ganador es el niño que saque una bola blanca. Si ambos sacan simultáneamente bolas de igual color, devuelven las bolas a sus cajas y el juego continúa. Eduardo afirma que el juego no es justo porque en la caja de Luis hay más bolas blancas que en la suya. ¿Cuál es tu opinión sobre lo que dice Eduardo?

Problema 2: María y Esteban juegan a lanzar un dado. María gana 1 confite si el dado sale 2 ó 3 ó 4 ó 5 ó 6 . Si resulta un 1, Esteban gana una cierta cantidad de confites. ¿Cuántos confites debe ganar Esteban cuando sale el 1, para que el juego sea justo o equitativo?

El primer problema es una adaptación de otro propuesto por Fischbein y Gazit (1984), y el segundo por Green (1982); ambos fueron empleados por Cañizares (1997). El problema 1 se resuelve mediante la comparación de probabilidades simples de un mismo suceso en dos experimentos con solo dos sucesos no 
equiprobables ("sacar una bola blanca" o "sacar una bola negra"). Debido a la composición de las urnas, se trata de un juego equitativo entre Eduardo y Luis; pues ambos tienen una probabilidad de ganar igual a $1 / 3$. Sin embargo, aparece un distractor que incita a realizar una comparación absoluta de los casos favorables, siendo esta una estrategia errónea. La respuesta correcta es indicar que Eduardo no tiene razón y los dos niños tienen igual probabilidad de ganar.

Se esperan argumentos basados en estrategias multiplicativas (correspondencia o razonamiento proporcional) aunque el significado personal varía de acuerdo con las prácticas de los estudiantes. Por ejemplo, sea $\mathrm{P}_{1}$ la práctica de una persona que indica que el juego es justo porque ambos niños tienen el doble de posibilidades de sacar una bola negra que de una blanca. De manera alternativa, se podría indicar que, aunque la caja de Luis contiene el triple de bolas blancas que la de Eduardo, también existe el triple de bolas negras, luego el juego es justo $\left(P_{2}\right)$. Mientras tanto una tercera solución podría ser indicar que Eduardo tiene razón al afirmar que no es juego justo; pues en la caja de Luis hay más bolas blancas que en la de Eduardo $\left(\mathrm{P}_{3}\right)$. Así, el significado en las dos primeras prácticas sería correcto, desde el punto de vista institucional; en tanto, la tercera evidencia un conflicto semiótico que se puede caracterizar por un razonamiento aditivo del sujeto al comparar probabilidades. En la Tabla 1 se presenta la configuración epistémica asociada al Problema 1.

TABLA 1

Configuración epistémica asociada al problema 1

\begin{tabular}{|c|c|c|}
\hline Tipos & $\begin{array}{l}\text { Objetos } \\
\text { matemáticos }\end{array}$ & Significado en la situación \\
\hline $\begin{array}{l}\text { Situación- } \\
\text { problema }\end{array}$ & $\begin{array}{l}\text { Decidir si un juego es } \\
\text { equitativo }\end{array}$ & Decidir si la ganancia esperada de los jugadores es la misma. \\
\hline \multirow{2}{*}{ Lenguajes } & $\begin{array}{l}\text { Sacar / devolver } \\
\text { Juego "justo" }\end{array}$ & $\begin{array}{l}\text { Reemplazamiento en una experiencia. } \\
\text { Igual posibilidad de ganancia. }\end{array}$ \\
\hline & $\begin{array}{l}\text { Números enteros y } \\
\text { comparativos }\end{array}$ & $\begin{array}{l}\text { Numerales }(10,20,30 \text { y } 60) \text { y sus relaciones comparativas ("más o menos que", } \\
\text { etc.) }\end{array}$ \\
\hline \multirow{6}{*}{ Conceptos } & Experimento aleatorio & El color en la bola extraída no se sabe a priori. \\
\hline & Espacio muestral & Idéntico en cada experimento aleatorio: $E=\{b, n\}$. \\
\hline & Evento o suceso & El evento evaluado es "sacar una bola blanca". \\
\hline & Probabilidad & $\begin{array}{l}\text { Definición clásica o Laplaciana: "fracción con denominador el número de } \\
\text { todos los casos posibles y con numerador el número de casos favorables al } \\
\text { suceso de interés" (Laplace, 1985/1814, p. 28). }\end{array}$ \\
\hline & Proporcionalidad & $\begin{array}{l}\text { Relación proporcional de bolas de cada color en las cajas de razón } 1 / 2 \text { (10b/20n } \\
\text { y 30b/60n). }\end{array}$ \\
\hline & Juego equitativo & Idéntica esperanza de ganancia de todos los jugadores. \\
\hline Proposiciones & Equiprobabilidad & $\begin{array}{l}\text { Si la razones de casos favorables y posibles de dos o más eventos son equiva- } \\
\text { lentes, los eventos son equiprobables. }\end{array}$ \\
\hline \multirow{4}{*}{ Procedimientos } & $\begin{array}{l}\text { Comparación } \\
\text { proporcional }\end{array}$ & En ambas cajas, por cada bola blanca hay dos bolas negras. \\
\hline & $\begin{array}{l}\text { Correspondencia entre } \\
\text { los elementos }\end{array}$ & $\begin{array}{l}\text { Aunque Luis tiene el triple de bolas blancas que Eduardo, también tiene el } \\
\text { triple de bolas negras. }\end{array}$ \\
\hline & $\begin{array}{l}\text { Cálculo de } \\
\text { probabilidades }\end{array}$ & Cociente entre el número de casos favorables y el número de casos posibles. \\
\hline & $\begin{array}{l}\text { Equivalencia de } \\
\text { ganancias }\end{array}$ & $\begin{array}{l}\text { Igualar las esperanzas de ganancia de todos los jugadores, si las probabilida- } \\
\text { des de los jugadores no son iguales. }\end{array}$ \\
\hline Argumento & Deductivo & $\begin{array}{l}\text { Los eventos simples son equiprobables por tener las cajas composiciones } \\
\text { proporcionales. }\end{array}$ \\
\hline
\end{tabular}

Fuente: elaboración propia. 
En el Problema 2 se pide encontrar el número de confites que deben recibir dos niños en un juego, de modo que se equipare la ganancia de los jugadores según la probabilidad que tienen de ganar. Para resolverlo, la persona tiene que darse cuenta de que las ganancias deben ser inversamente proporcionales a la probabilidad de ganar de cada jugador; es decir, como Esteban tiene una probabilidad de perder cinco veces mayor que María, si ella gana un confite, entonces Esteban debería obtener cinco confites para que el juego sea justo. La configuración epistémica del problema se presenta en la Tabla 2.

TABLA 2

Configuración epistémica asociada al problema 2

\begin{tabular}{|c|c|c|}
\hline Tipos & $\begin{array}{l}\text { Objetos } \\
\text { matemáticos }\end{array}$ & Significado en la situación \\
\hline $\begin{array}{l}\text { Situación- } \\
\text { problema }\end{array}$ & $\begin{array}{l}\text { Decidir el número de } \\
\text { confites que debe ganar } \\
\text { un jugador para que el } \\
\text { juego sea equitativo }\end{array}$ & Igualar la esperanza matemática de los dos jugadores \\
\hline \multirow{2}{*}{ Lenguajes } & $\begin{array}{l}\text { Lanzar un dado } \\
\text { Juego "justo" }\end{array}$ & $\begin{array}{l}\text { Experimento aleatorio. } \\
\text { lgual ganancia esperada. }\end{array}$ \\
\hline & Números enteros & Numerales (1 a 6) \\
\hline \multirow{6}{*}{ Conceptos } & Experimento aleatorio & El número que saldrá en el dado no se conoce a priori \\
\hline & Espacio muestral & Conjunto de resultados. $E=\{1,2,3,4,5,6\}$ \\
\hline & Evento o suceso & El evento evaluado es "sacar un 1". \\
\hline & Probabilidad & $\begin{array}{l}\text { Definición clásica o Laplaciana: "fracción con denominador el número de } \\
\text { todos los casos posibles y con numerador el número de casos favorables al } \\
\text { suceso de interés" (Laplace, 1985/1814, p. 28). }\end{array}$ \\
\hline & $\begin{array}{l}\text { Proporcionalidad } \\
\text { inversa }\end{array}$ & $\begin{array}{l}\text { Debe haber proporcionalidad inversa entre la probabilidad y la ganancia de } \\
\text { cada jugador }\end{array}$ \\
\hline & Juego equitativo & Idéntica esperanza de ganancia de todos los jugadores. \\
\hline Proposiciones & Equiprobabilidad & Todos los números del dado son equiprobables. \\
\hline \multirow{2}{*}{ Procedimientos } & $\begin{array}{l}\text { Cálculo de } \\
\text { probabilidades }\end{array}$ & Cociente entre el número de casos favorables y el número de casos posibles. \\
\hline & $\begin{array}{l}\text { Equivalencia de } \\
\text { ganancias }\end{array}$ & $\begin{array}{l}\text { Igualar las esperanzas de ganancia de todos los jugadores, si las probabilida- } \\
\text { des de los jugadores no son iguales. }\end{array}$ \\
\hline Argumento & Deductivo & $\begin{array}{l}\text { Si un jugador tiene cinco veces más probabilidad, el otro debe tener cinco } \\
\text { veces más ganancia en cada juego. }\end{array}$ \\
\hline
\end{tabular}

Fuente: elaboración propia.

\section{Metodología de análisis}

Se llevó a cabo un análisis cuantitativo y cualitativo de las respuestas a los dos problemas planteados para evaluar los significados personales sobre la noción de juego equitativo, mediante el análisis de sus prácticas al resolver dichos problemas. Según Cañizares y Batanero (1997, p. 103), se asume que "la simple consideración de las respuestas correctas nos puede llevar a conclusiones engañosas sobre el razonamiento probabilístico de los alumnos"; por lo tanto, se analizaron los argumentos formulados por el estudiantado para identificar aquellos concordantes con los significados institucionales y detectar posibles conflictos semióticos (Godino, Batanero y Font, 2007; 2019).

Más concretamente, se llevó a cabo un análisis de contenido (Zapico, 2007) de las respuestas escritas para profundizar en ellas; pues según Krippendorff (2013), este método nos permite establecer categorías de 
análisis que emergen de modo objetivo como resultado del análisis sistemático realizado. Este análisis se complementa con información numérica, que se muestra mediante porcentajes para la variable cuantitativa "grado de corrección".

Las variables consideradas fueron la respuesta al problema y el argumento utilizado. Las respuestas a los problemas se clasificaron en correctas, si se indica en el primer problema que el juego es equitativo y si se calcula en forma correcta la ganancia esperada en el segundo problema, e incorrecta si no se llega a estas soluciones. En las soluciones correctas de ambos problemas se tienen en consideración los objetos matemáticos incluidos en las configuraciones epistémicas explicitadas en las tablas 1 y 2 . Los argumentos se clasificaron como correctos, parcialmente correctos e incorrectos y en la sección de resultados se muestran ejemplos de cada una de estas categorías.

\section{DISCUSIÓN DE RESULTADOS}

Los resultados muestran que el Problema 2 tuvo mayor porcentaje de respuestas correctas que el Problema 1 (32,7\% vs. 16,4\%) y esta diferencia se explica por una mayor dificultad en el Problema 1; pues el distractor "Eduardo afirma que el juego no es justo porque en la caja de Luis hay más bolas blancas que en la suya" pudo haber inducido a emplear estrategias aditivas para comparar las probabilidades. Por tanto, no se utiliza la comparación proporcional o la correspondencia entre los elementos de las urnas asociadas a esta configuración epistémica (Tabla 1).

Respecto a los resultados en investigaciones anteriores, con estudiantado de la misma edad (11-12 años), en ambos problemas los porcentajes de respuestas correctas en el presente estudio fueron bastante inferiores. Con respecto al Problema 1, en Cañizares (1997) se obtuvo 63,8\% de respuestas correctas en su primer estudio y $32,5 \%$ en el segundo, y en Fischbein y Gazit (1984) se obtuvo 50,4\% de respuestas correctas en estudiantado que no tuvo instrucción previa y 53,0\% en los que sí la tuvieron. En el Problema 2, el porcentaje de respuestas correctas obtenido en el primer estudio de Cañizares (1997) fue 45,1\%, en su segundo estudio el 56,8\% y en Green (1982) fue del 46,0\%; en definitiva, más de diez puntos porcentuales por encima de los resultados de este trabajo.

\section{Análisis de argumentos}

Con la finalidad de identificar los conflictos semióticos y las creencias de tipo subjetivo que intervienen en la asignación de probabilidades y pueden incidir en los significados personales sobre probabilidad y el concepto de "juego justo", se llevó a cabo un estudio de los argumentos que se clasificaron en correctos, parcialmente correctos e incorrectos.

En relación con la solución del Problema 1 se consideraron correctos los argumentos asociados a estrategias de correspondencia proporcional (Tabla 1) para comparar las probabilidades de los dos jugadores. En estas estrategias se establece un criterio de proporcionalidad entre los casos favorables y desfavorables de un jugador para compararlo con esa misma relación en el otro jugador. Este tipo de argumento se observa en los dos ejemplos siguientes:

E1: "Yo creo que tienen exactamente las mismas probabilidades porque Eduardo tiene 10 (bolas blancas) lo que es la mitad de 20 (bolas negras) y Luis tiene 30 que es la mitad de 60; entonces Eduardo está incorrecto". 
E2: "Mi opinión sobre lo que dice Eduardo es que tiene un poco de razón porque en la caja de Luis sí hay más bolas blancas que en la suya, pero los dos niños tienen el doble de bolas negras que bolas blancas en cada una de sus cajas".

Esta estrategia para comparar fracciones fue descrita por Noelting (1980a, 1980b), quien diferencia dos tipos: en el primer tipo, que denominó "entre", se comparan los términos de una fracción con los de la otra; en el segundo tipo (estrategia "intro") se comparan numerador y denominador de una misma fracción para establecer una razón y luego esta razón se compara con la correspondiente en la segunda fracción.

En los ejemplos analizados, E1 utiliza una estrategia "intro", donde se establece la razón (1:2) de la fracción correspondiente a Eduardo, y por tanto, usa un procedimiento de correspondencia entre los elementos (Tabla 1), para posteriormente compararla con la razón correspondiente a Luis. Por otro lado, aunque E2 primero realiza una comparación del número absoluto de casos favorables (inducido por el distractor antes mencionado), luego establece la razón de bolas negras respecto a las blancas (2:1); es decir, una comparación proporcional (Tabla 1), para responder en forma correcta a la pregunta.

Se consideran parcialmente correctos algunos argumentos que muestran conflictos semióticos, como se muestra en los siguientes ejemplos:

E3: “No porque él tiene 60 negras y Eduardo 20 si multiplicamos por 3 las de Eduardo va a salir la misma cantidad de Luis".

E4: “Eduardo tiene y no tiene razón; tiene razón porque Luis tiene 20 bolas más que Eduardo pero Luis tiene más bolas negras que blancas, entonces ambos tienen la misma probabilidad".

En el primer ejemplo, E3 se centra en la diferencia entre los casos desfavorables (3 frente a 1) y, en consecuencia, no usa ninguno de los procedimientos explicitados en la Tabla 1, ni tampoco la regla de Laplace, aunque implícitamente pareciera que considera los casos favorables; sin embargo, muestra un conflicto semiótico al no indicarlos en su argumento al comparar probabilidades y otro también al realizar comparaciones en forma aditiva; por lo tanto, el argumento no es por completo correcto.

Por su parte, E4 muestra también el conflicto semiótico consistente en usar una estrategia aditiva; pues compara los casos favorables en los dos jugadores y halla su diferencia y no su razón (solo indica que hay más casos favorables que desfavorables); sin embargo, lo consideramos parcialmente correcto porque sí indica que los dos jugadores tienen igual probabilidad. Este sujeto tampoco usa la regla de Laplace ni ninguno de los procedimientos correctos detallados en la Tabla 1.

También existen, argumentos correctos o parcialmente correctos, pero con respuestas incorrectas, por ejemplo:

E5: "Eduardo tiene razón porque la probabilidad de Eduardo que saque una bola blanca no es tan eficiente; en cambio Luis tiene un poco de ventaja. Pero si lo ven bien se atienen a la suerte, porque las negras es la mitad de la blanca, 10 blancas y 20 negras o 30 blancas y 60 negras".

E6: "Creo que debería ganar 6 confites porque él tiene menos probabilidades de ganar".

Aunque la respuesta de E5 (asociada al Problema 1) es incorrecta, en su argumento hay evidencia de que percibe la relación de equivalencia (1/2) entre la razones de bolas negras y blancas de Eduardo y Luis y por tanto la equiprobabilidad de los dos jugadores, y la proporcionalidad de las urnas, elementos explicitados en la Tabla 1. Asimismo, la respuesta de E6 al Problema 2, aunque es incorrecta, usa un 
argumento parcialmente correcto al notar la relación inversa básica (Tabla 2, aunque no identifica la proporcionalidad) entre el número de casos favorables y de confites que ganarían las personas.

Por otra parte, se consideraron argumentos incorrectos los que giraron en torno a lo que planteaba el distractor, que indicaría un conflicto semiótico consistente en suponer que hay más probabilidad siempre que haya más casos favorables, de modo que no se usan los procedimientos correctos explicitados en las Tablas 1 y 2.

En general, estos sujetos compararon solo el valor absoluto del número de casos favorables (Ver E7) o desfavorables (Ver E8). Este conflicto semiótico también fue encontrado en Cañizares et al. (1999), donde los sujetos únicamente suponían que menos casos favorables indican menor probabilidad.

E7: "Sí hay más probabilidad de que Luis gane ya que tiene más bolas blancas que Eduardo".

E8: “En mi opinión Eduardo tiene más ventaja que desventaja, debido a que Luis tiene 40 bolas negras más que Eduardo y solo tiene 20 bolas blancas más".

Otros conflictos semióticos que afloraron en este problema fueron suponer que el juego sería equitativo solo si se juega exactamente con el mismo número de bolas de cada color, o indicar que el juego no es equitativo porque un jugador tiene en total 90 bolas y el otro solo 30; es decir, porque no tienen el mismo número de casos posibles. En estas situaciones, se añaden elementos que no forman parte de las configuraciones epistémicas (Tabla 1) porque son incorrectos a nivel matemático.

Encontramos mayor dificultad al argumentar la respuesta al Problema 2, donde no se hallaron argumentos completamente correctos, aunque cabe mencionar que una proporción importante de estudiantado identifica la mayor probabilidad de María para ganar. De este modo, se han considerado correctas las respuestas que indican que Esteban debe recibir cinco confites para que el juego sea justo, aunque no lo justifiquen o su justificación sea incorrecta, puesto que llegaron a equiparar la ganancia. Estos sujetos muestran una intuición correcta sobre la idea de esperanza matemática (Schlottmann y Anderson, 1994), aunque presenten algún conflicto semiótico en sus argumentaciones; como por ejemplo se muestra en el argumento de $E 9$, quien no asume la independencia en las partidas del juego: $E 9$ : "Para que el juego sea justo Esteban debería ganar lo que María ganaría si tirara el dado 5 veces con su probabilidad (5 confites)".

Se considera parcialmente correcto el argumento si se identifica la relación inversa entre la probabilidad y ganancia, aunque haya un error en la obtención de la probabilidad de ganar cada jugador, como el caso de E10. En este, sin embargo, el estudiante tiene una respuesta correcta, aunque no empleó alguno de los procedimientos explicitados en la Tablas 2: E10: "Tiene que ganar 5 porque María tiene el 75\% más de probabilidad de ganar."

Los argumentos incorrectos en el Problema 2 se deben a conflictos semióticos, los cuales fueron indicar que el juego sería equitativo si también Esteban ganara un confite al salir el 1; es decir, que los jugadores debieran jugar al mismo número; calcular en forma incorrecta el número de confites (por ejemplo, 2 o 6 o bien especificar que hay que darle más, sin especificar cuántos). En todos estos casos se observa dificultad con la proporcionalidad y añaden elementos no contemplados en la configuración epistémica expuesta en la Tabla 2, que son matemáticamente incorrectos.

En la Tabla 3 se presenta un resumen de los resultados del análisis de los argumentos en cada problema; también muestra la proporción de respuestas en cada uno, según el grado de corrección. 
TABLA 3

Porcentaje de respuestas y argumentos en los problemas propuestos (P1: Problema 1; P2: Problema 2)

\begin{tabular}{|c|c|c|c|c|c|c|c|c|c|c|}
\hline \multirow{3}{*}{$\begin{array}{l}\text { Tipo de } \\
\text { respuesta }\end{array}$} & \multicolumn{10}{|c|}{ Argumento' } \\
\hline & \multicolumn{2}{|c|}{ Correcto } & \multicolumn{2}{|c|}{$\begin{array}{l}\text { Parcialmente } \\
\text { correcto }\end{array}$} & \multicolumn{2}{|c|}{ Incorrecto } & \multicolumn{2}{|c|}{ No argumenta } & \multicolumn{2}{|c|}{ Total $^{2}$} \\
\hline & P1 & P2 & P1 & P2 & $\mathrm{P} 1$ & P2 & P1 & P2 & P1 & P2 \\
\hline Correcta & 22,2 & 22,2 & 66,7 & 38,8 & 11,1 & 16,7 & & 22,2 & 16,4 & 32,7 \\
\hline Incorrecta & 2,2 & & 6,5 & 27,0 & 87,0 & 56,8 & 4,3 & 16,2 & 83,6 & 67,3 \\
\hline
\end{tabular}

'Porcentaje respecto al total de la fila; ${ }^{2}$ Porcentaje respecto al total de sujetos.

Fuente: elaboración propia.

Se observa que el porcentaje de argumentos correctos cuando la respuesta fue correcta es el mismo en ambos problemas (22,2\%); mientras la relación de respuestas correctas con argumentos correctos o parcialmente correctos no es la misma y los resultados muestran la mayor dificultad ante la argumentación en el Problema 2 (88,9\% de argumentos correctos o parcialmente correctos en el Problema 1 y $61,0 \%$ en el Problema 2).

Cabe señalar que Cañizares (1997) obtuvo para este primer problema un 26,6\% de argumentos pertinentes asociados con la estrategia de correspondencia, frente al $21,9 \%$ obtenido en el presente estudio asociado a dicha estrategia, lo cual revela la dificultad del problema. La frase que sugiere que el juego no es justo, pues en la caja de Luis hay más bolas blancas que en la de Eduardo ha sido un distractor en el Problema 1, lo cual influye en los argumentos y provoca que el nivel de dificultad aumente. Sin embargo, esta tarea se considera apropiada para la etapa de desarrollo del razonamiento proporcional en edad promedio de 10,5 años, según Noelting (1980a y 1980b), debido a la composición de las bolas en las cajas.

Por último, cabe destacar que, aunque el estudiantado había recibido instrucción en el tema de probabilidades, no había trabajado este tipo de tareas sobre juego equitativo. A pesar de ello, parte de la muestra resolvió con éxito los problemas y ofrecieron argumentos pertinentes; por lo tanto, los resultados obtenidos serían producto de las intuiciones primarias que haya adquirido el estudiantado y no debido a la enseñanza recibida.

\section{SÍNTESIS Y REFLEXIONES FINALES}

Consideramos que el trabajo presentado es valioso; pues brinda conocimiento sobre los significados personales del estudiantado costarricenses acerca de la noción de juego equitativo y esta información siempre es útil al profesorado de cara a identificar diversos conflictos que se presentan en el estudio del tema. Las principales conclusiones sobre este significado se describen en los próximos párrafos.

En primer lugar, los participantes en general mostraron una adecuada comprensión de la noción de juego equitativo, aunque se presentaron algunos conflictos semióticos como considerar que para ser equitativo todos los jugadores han de jugar al mismo resultado o no tener en cuenta la independencia de ensayos sucesivos. Más difícil para ellos fue la comparación de probabilidades, de nuevo se mostraron conflictos consistentes en considerar solo los casos favorables, únicamente los desfavorables o la diferencia entre ellos en dicho cálculo (estrategias aditivas).

Por otra parte, presentan grandes dificultades al justificar la equiparación de ganancia (Problema 2) según la esperanza de ganar de cada jugador, lo cual es debido a una falta de desarrollo de razonamiento 
proporcional. Una parte de la muestra asigna igualdad de ganancias y manifiesta argumentos asociados al sesgo de equiprobabilidad (Lecoutre, 1992), resultados similares a los documentados en Cañizares et al. (1999).

Aunque a priori se esperaba encontrar diferencias respecto a los resultados obtenidos con sujetos de la misma edad en investigaciones previas (Cañizares, 1997; Fischbein y Gazit, 1984; Green, 1982), puesto que el estudiantado en la muestra sí había recibido instrucción en probabilidad, estas expectativas no se cumplieron. Se preveía obtener mejores resultados en relación con dichos estudios y se esperaba una influencia positiva de la instrucción en las intuiciones (Fischbein y Gazit, 1984; Fischbein, Pampu y Minzat, 1967). La explicación de los resultados obtenidos es que, aunque los sujetos de la muestra han estudiado algunos contenidos probabilísticos, las tareas matemáticas propuestas en este estudio no han sido habituales para ellos durante su instrucción.

Como síntesis, aunque estos problemas hayan resultado difíciles, es importante enfrentar al estudiantado a este tipo de situaciones según el enfoque metodológico de resolución de problemas planteado por el currículo costarricense, el cual indica que: "la filosofía a seguir en el aula varía a favor de acentuar acciones cognitivas de mayor nivel" (MEP, 2012, p. 32). Estos problemas podrían ser empleados como punto de partida en la acción educativa para generar indagación y discusión entre el estudiantado. Se recomienda incorporar en las lecciones costarricenses y en los libros de texto utilizados, tareas matemáticas en las cuales se formule y trabaje la equitatividad de un juego, no solo en el caso donde todos los jugadores tienen la misma probabilidad de ganar y obtengan el mismo premio, sino también explorar situaciones donde las probabilidades de los jugadores sean diferentes y se deban equiparar los premios igualando las esperanzas de ganancia.

El trabajo aporta ideas para continuar la indagación sobre la comprensión del juego equitativo, planteando otros juegos basados en experimentos compuestos o en otros dispositivos aleatorios diferentes, como ruletas o cartas. Se anima a otros investigadores a continuar este trabajo; pues hay carencia de investigaciones respecto a razonamiento probabilístico con el estudiantado costarricense. Se espera continuarlo con muestras más grandes que, además, consideren diferentes niveles educativos, lo cual podría brindar insumos valiosos para la adecuación de propuestas curriculares para diferentes edades. Otra posibilidad es plantear diseños instruccionales basados en los juegos de azar en que se introduzcan explícitamente en el aula las ideas estocásticas fundamentales de Gal (2005).

Por otro lado, si se toma en cuenta que en el contexto de implementación curricular costarricense, una parte del profesorado siente inseguridad al enseñar la probabilidad debido a una débil formación conceptual y didáctica en el tema (Alpízar et al., 2012; Alpízar, Chavarría y Oviedo, 2015); se plantea la necesidad de una investigación más profunda que considere diferentes variables a la luz de la mediación pedagógica que se realiza en el aula en torno al razonamiento probabilístico. En diversas investigaciones se sugiere reforzar la formación del profesorado, tanto en probabilidad como en su didáctica, debido a las dificultades que se observan en este tema (Azcárate, 1995; Mohamed y Ortiz, 2012; Ortiz, Batanero y Contreras, 2012). Por tanto, dicha formación podría tener en cuenta los componentes del conocimiento del profesorado establecidas en el modelo CCDM (Godino, Giacomone, Batanero y Font, 2017), así como la teoría de idoneidad didáctica, lo cual podría también orientar la elaboración de propuestas didácticas de enseñanza y aprendizaje de la probabilidad (Beltrán-Pellicer, Godino y Giacomone, 2018).

De este modo, estos resultados pueden ser útiles a formadores para el diseño de procesos de capacitación docente y cursos de formación del profesorado, donde se reflexione sobre las demandas cognitivas de las tareas planteadas al estudiantado, de sus formas de razonamiento y posibles conflictos semióticos, y de cómo estos elementos podrían orientar la planificación educativa.

Agradecimiento

Proyecto PID2019-105601GB-I00 / AEI / 10.13039/501100011033 y Grupo FQM126 (Junta de Andalucía). 


\section{REFERENCIAS}

Azcárate, P. (1995). El conocimiento profesional de los profesores sobre las nociones de aleatoriedad y probabilidad. Su estudio en el caso de la educación primaria. Tesis doctoral inédita. Cádiz: Universidad de Cádiz.

Alpízar, M., Barrantes, J., Bolaños, H., Céspedes, M., Delgado, E., Freer, D., Padilla, E., y Víquez, M. (2012). Aspectos relevantes sobre la formación docente en I y ll ciclos en los temas Probabilidad y Estadística. EDUCARE, 16(2):113-129.

Alpízar, M., Chavarría, L. y Oviedo, K. (2015). Percepción de un grupo de docentes de l y ll ciclo de educación general básica de escuelas públicas de Heredia sobre los temas de estadística y probabilidad. Actualidades Investigativas en Educación, 15(1):1-23. DOI: dx.doi.org/10.15517/aie.v15i1.17728

Australian Curriculum, Assessment and Reporting Authority (ACARA). (2013). The Australian curriculum: Mathematics. Sidney, NSW: Author.

Batanero, C. (2005). Significados de la probabilidad en la educación secundaria. Revista Latinoamericana de Matemática Educativa, 8(3):247-264.

Batanero, C., Henry, M. y Parzysz, B. (2005). The nature of chance and probability. En G. A. Jones (Ed.), Exploring probability in school: Challenges for teaching and learning. New York, USA: Springer.

Batanero, C., Ortiz, J., Gómez, E. y Gea, M. M. (2019). Les jeux équitables comme contexte pour l'enseignement des probabilités et la formation des enseignants. En V. Martin, M. Thibault y L. Theis (Eds.), Enseigner les premiers concepts de probabilités. Québec: Presses de l'Université de Québec.

Bayless, S. y Schlottmann, A. (2010). Skill-Related Uncertainty and Expected Value in 5-to 7-Year-Olds. Psicologica: International Journal of Methodology and Experimental Psychology, 31(3):677-687.

Beltrán-Pellicer, P., Godino, J. D. y Giacomone, B. (2018). Elaboración de Indicadores Específicos de Idoneidad Didáctica en Probabilidad: Aplicación para la Reflexión sobre la Práctica Docente. Bolema, 32(61):526-548.

Bisquerra, R. (1989). Métodos de investigación educativa. Barcelona: P.P.U.

Cañizares, M. J. (1997). Influencia del razonamiento proporcional y combinatorio y de creencias subjetivas en las intuiciones probabilísticas primarias. Granada: Tesis Doctoral. Universidad de Granada.

Cañizares, M. y Batanero, C. (1997). Influencia del razonamiento proporcional y de las creencias subjetivas en la comparación de probabilidades. UNO, 14:99-114.

Cañizares, M., Batanero, C., Serrano, L. y Ortiz, J. (1999). Comprensión de la idea de juego equitativo en los niños. Números, 37:37-55.

Cerrón, W. (2019). La investigación cualitativa en educación. Horizonte de la Ciencia, 9(17):1-8. DOI: 10.26490/uncp.horizonteciencia.2019.17.510

Fischbein, E. (1975). The intuitive sources of probabilistic thinking in children. Dordrecht: Reidel.

Fischbein, E. (1987). Intuition in science and mathematics. Dordrecht: Reidel.

Fischbein, E. y Gazit, A. (1984). Does the teaching of probability improve probabilistic intuitions? Educational Studies in Mathematics, 15(1):1-24.

Fichbein. E., Pamput, E. y Minzat, I. (1967). The child's intuition of probability. Enfance, 2:193-280.

Gal, I. (2005). Towards "probability literacy" for all citizens: Building blocks and instructional dilemmas. En G. Jones (Ed.), Exploring probability in school. Boston, MA: Springer.

Gil, J., León, J. y Morales, M. (2017). Los paradigmas de investigación educativa, desde una perspectiva crítica. Conrado, 13(58):72-74. 
Godino, J. y Batanero, C. (1994). Significado institucional y personal de los objetos matemáticos. Recherches en Didactiques des Mathématiques, 14(3): 325-355.

Godino, J. Batanero, C. y Font, V. (2007). The ontosemiotic approach to research in mathematics education. ZDM. The International Journal on Mathematics Education, 39(1-2):127-135.

Godino, J. D., Batanero, C. y Font, V. (2019). The onto-semiotic approach: Implications for the prescriptive character of didactics. For the Learning of Mathematics, 39(1):38-43.

Godino, J. D., Giacomone, B., Batanero, C. y Font, V. (2017). Enfoque ontosemiótico de los conocimientos y competencias del profesor de matemáticas. Bolema, 31(57):90-113.

Green, D. R. (1982). Probability concepts in school pupils aged 11-16 years. Ph. Dissertation. Reino Unidos: University of Loughborough.

Hernández-Solís, L. A., Batanero, C., Gea, M. M. y Álvarez-Arroyo, R. (2021). Comparing probabilities in urns: A study with primary school students. Uniciencia, 35(2):1-19.

Jones, G., Langrall, C., y Mooney, E. (2007). Research in probability: responding to classroom realities. En F. Lester (Ed.), Second handbook of research on mathematics teaching and learning (vol. 2,). Greenwich: CT: Information Age Publishing y NCTM.

Krippendorff, K. (2013). Content analysis: an introduction to its methodology. London, Sage.

Laplace, P. S. (1985). Ensayo filosófico sobre las posibilidades. Madrid: Alianza Editorial. (Trabajo original publicado en 1814).

Lecoutre, M. P. (1992). Cognitive models and problem spaces in "purely random" situations. Educational Studies in Mathematics, $23: 557-568$.

Lidster, S. T., Watson, J. M., Collis, K. F. y Pereira-Mendoza, L. (1996). The relationship of the concept of fair to the construction of probabilistic understanding. En Clarkson, P. C. (Ed.), Technology in Mathematics Education, Proceedings of the Nineteenth Annual Conference of the Mathematics Education Research Group of Australasia, Melbourne. Sydney: MERGA.

Ministerio de Educación, Cultura y Deporte (MECD). (2014). Real Decreto 1105/2014, de 26 de diciembre, por el que se establece el currículo básico de la Educación Secundaria Obligatoria y del Bachillerato. Madrid: Autor.

Ministerio de Educación Pública (MEP). (2012). Programas de Estudio de Matemáticas. I, II Y III Ciclos de la Educación General Básica y Ciclo Diversificado. San José: Autor.

Mohamed, N. y Ortiz, J. (2012). Evaluación de conocimientos de profesores en formación sobre el juego equitativo. Números, 80:103-117.

National Council of Teachers of Mathematics (NCTM). (2000). Principles and standards for school mathematics. Reston, VA: The Council.

Nilsson, P. y Li, J. (2015). Teaching and learning of probability. En S. J. Cho (Ed.). The Proceedings of the 12th International Congress on Mathematical Education: Intellectual and Attitudinal Challenges. New York: Springer.

Noelting, G. (1980a). The development of proportional reasoning and the ratio concept. Part I. Diferentiation of stages. Educational Studies in Mathematics, 11(2):217-253.

Noelting, G. (1980b). The development of proportional reasoning and the ratio concept. Part II. Problem structure at successive stages: Problem solving strategies and the mechanism of adaptive restructuring. Educational Studies in mathematics, 11(3):331-363.

Ortiz, J., Batanero, C. y Contreras, J. (2012). Conocimiento de futuros profesores sobre la idea de juego equitativo. Revista Latinoamericana de Investigación en Matemática Educativa, 15 (1):63-9. 
Piaget, J., e Inhelder, B. (1951). La genése de l'idée de hasard chez l'enfant. Paris: Presses Universitaires de France.

Pino, G. y Estrella, S. (2012). Educación estadística: relaciones con la matemática. Pensamiento Educativo. Revista de Investigación Educacional Latinoamericana, 49(1):53-64.

Peard, R. (1990). Gambling and ethnomathematics in Australia. En Booker, G., Cobb, P. y Mendicutti, T. (Eds). Proceedings of the XIV PME Conference (V.2,). México: PME Group.

Santillana (2019a). Matemática 5. (1a. ed.). San José, Costa Rica: Editorial Santillana.

Santillana (2019b). Matemática 5: Cuaderno de actividades. (1a. ed.). San José, Costa Rica: Editorial Santillana.

Schlottmann, A. y Anderson, N. H. (1994). Children's judgements of expected value. Developmental Psychology, 30 (1):56-66.

Watson, J. y Collis, K. F. (1994). Multimodal functioning in understandi chance and data concepts. En Ponte, J. P. y Matos, J. P. (Eds), Proceedings of the XVIII International Conference for the Psychology of Mathematics Education. Portugal: Universidad de Lisboa.

Zapico, M. (2007). Interrogantes acerca de análisis de contenido y del discurso en los textos escolares. En MINEDUC (Ed.), Primer Seminario Internacional de Textos Escolares (SITE 2006) (pp. 149-155). Santiago: MINEDUC. 